\title{
Quantum size effects in quasi-free-standing Pb layers
}

\author{
J. H. Dil, ${ }^{1}$ T. U. Kampen, ${ }^{1}$ B. Hülsen, ${ }^{1}$ T. Seyller, ${ }^{2}$ and K. Horn ${ }^{1}$ \\ ${ }^{1}$ Fritz-Haber-Institut der Max-Planck-Gesellschaft, Berlin, Germany \\ ${ }^{2}$ Lehrstuhl für Technische Physik, Universität Erlangen-Nürnberg, Erlangen, Germany
}

(Received 13 February 2007; published 11 April 2007)

\begin{abstract}
Quantum well states in thin metal films are strongly influenced by the electronic and geometric structure of the underlying substrate. This impedes a comparison to $a b$ initio calculations generally based on free-standing films. Here we show that $\mathrm{Pb}$ films deposited on single-crystalline, epitaxial graphite are an excellent approximation to free-standing metal films. We find $\mathrm{Pb}$ islands of four monolayer height to be most stable, followed by other even-numbered islands, in excellent agreement with theoretical predictions for the formation of preferred island heights.
\end{abstract}

DOI: 10.1103/PhysRevB.75.161401

PACS number(s): 79.60.Dp, 73.21.Fg, 68.55.Jk, 71.15.-m

The electronic structure and morphology of ultrathin metal films is a subject of intense scientific and technological interest. In such films, the thicknesses are comparable to the electron coherence length, and quantum well states (QWSs) may form due to electron confinement in the direction normal to the surface. ${ }^{1,2}$ Many physical properties therefore show a strong dependence on layer thickness, and such quantum size effects (QSEs) have been found to induce, and thus to be reflected in, the oscillation with the thickness of macroscopic properties such as sign and magnitude of the Hall effect, ${ }^{3,4}$ reactivity and absorption, ${ }^{5}$ the magnitude of the superconducting transition temperature, ${ }^{6,7}$ and details of the growth morphology. ${ }^{8}$ Moreover, the study of electron confinement in metal films has contributed to an understanding of basic solid-state physics phenomena such as electronphonon coupling. ${ }^{9}$ One of the striking consequences of electron confinement is its influence on film growth morphology. Zhang and co-workers have predicted the formation of "critical" and "magic" island heights from a theoretical study of the variation of the total energy with coverage. Their "electronic growth" model ${ }^{10}$ has been corroborated by a number of experiments. Such growth morphologies were found to depend sensitively on details of the interface structure. ${ }^{11}$ Scanning tunnelling microscopy (STM) results from the $\mathrm{Pb} / \mathrm{Cu}(111)$ system $^{12}$ show a variety of preferred island heights, partly depending on the step density of the substrate. Direct proof of the interplay between electronic structure and morphology is derived from the observation of quantum well states in angle-resolved photoemission spectroscopy (ARPES). Here an assignment of specific island thicknesses can be obtained through an analysis of photoemission peaks due to quantum well states on the basis of the so-called phase accumulation model. ${ }^{13}$

Since the substrate apparently has a strong influence on the development of specific preferred thicknesses, it appears preferable to study metal film growth on a material which exhibits a very weak interaction with the overlayer. This is also advantageous because calculations of metal film behavior rely on free-standing slab models, since the interface of the (in general incommensurate) substrate/film lattices is difficult to model. Graphite is a material that is known to interact weakly with many metals; moreover, the density of states near the Fermi level is low over a relatively wide energy and momentum range. Hence graphite appears as a good substrate candidate for such studies.

Highly ordered pyrolytic graphite (HOPG) has frequently been used as a substrate in studies. However, this material presents surfaces with many rotated domains-i.e., the equivalent of a polycrystalline material in two dimensions. This is a disadvantage for wave-vector-resolved studies of electronic states. Here we use a recently developed procedure based on the formation of a single-crystalline graphite surface from silicon carbide. ${ }^{14}$

The sample used for the experiments was on-axis-oriented $6 \mathrm{H}$-SiC(0001) ( $\mathrm{Si}$ face) with a doping concentration of $1 \times 10^{18} \mathrm{~cm}^{-3}$. In order to achieve a surface free from polishing damage it was plasma etched in 1 bar hydrogen at $1550{ }^{\circ} \mathrm{C}$ for $30 \mathrm{~min} .{ }^{15} \mathrm{By}$ repeated annealing for several minutes at $1550{ }^{\circ} \mathrm{C}$, first oxygen and then silicon from the top layers are evaporated and a smooth, single-domain graphite sheet of several monolayers thickness with large terrace sizes remains. At the centre of the 2D Brillouin zone the (0001) surface has no bands down to $4 \mathrm{eV}$ below the Fermi level, and in the $\Gamma M$ direction no bands will come closer to $E_{\mathrm{F}}$ than $3 \mathrm{eV} .^{16}$

Lead was evaporated on this substrate from a watercooled Knudsen cell, while the substrate was held at $100 \mathrm{~K}$. Angle-resolved photoemission experiments were performed at the $10-\mathrm{m}$ normal incidence monochromator beamline at BESSY II in Berlin with a PHOIBOS 100 analyzer (Specs $\mathrm{GmbH})$. This analyzer allows for the simultaneous recording of kinetic energy and exit angle of the photoemitted electrons. Within the measurement conditions an energy and angular resolution better than $80 \mathrm{meV}$ and $0.2^{\circ}$, respectively, are reached. A photon energy of $24 \mathrm{eV}$ was used, because at this energy $\mathrm{Pb}$ has a direct transition just below the Fermi level, ${ }^{17}$ such that the cross section for photoemission from quantum well states is enhanced. ${ }^{18}$

The band structure calculations were performed within the generalized gradient approximation ${ }^{19}$ to density functional theory, using the highly accurate all-electron fullpotential linearized augmented plane-wave (FP-LAPW) method implemented in the WIEN $2 \mathrm{k}$ code. ${ }^{20}$ The muffin-tin radius was set to 2.5 a.u.; in the interstitial part, a plane-wave expansion cutoff of $13 \mathrm{Ry}$ was used. The $\mathrm{Pb}$ films were simulated using a supercell containing two or four atomic 


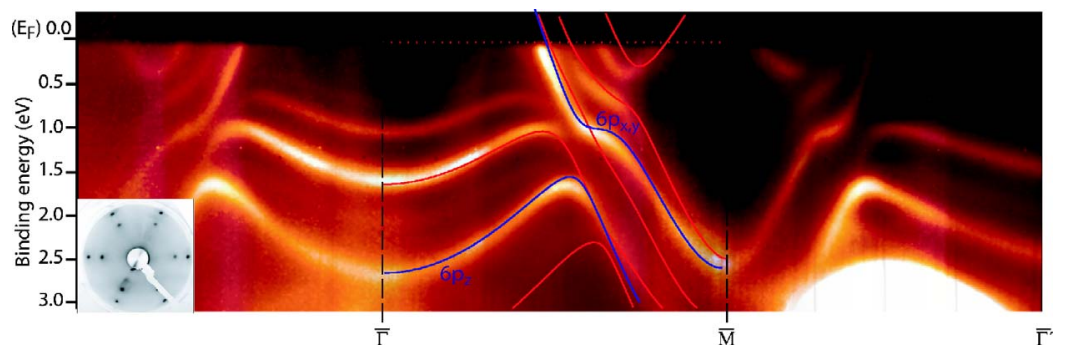

FIG. 1. (Color online) Cut through the SBZ for $2 \mathrm{ML}$ of $\mathrm{Pb}$ on graphitized $\mathrm{SiC}$. The lines represent the DFT calculations for a 2-ML- [blue (dark gray)] and a 4-ML- [red (light gray)] thick free-standing $\mathrm{Pb}$ slab, with spin-orbit coupling taken into account. (Inset) LEED image obtained at $90 \mathrm{eV}$ after deposition of $2 \mathrm{ML}$ of $\mathrm{Pb}$.

layers and a $15 \AA$ vacuum region, and all internal atomic coordinates were relaxed until the forces on the nuclei were smaller than $2 \mathrm{mRy} / \mathrm{a} . \mathrm{u}$. Within this approach, only the core electrons are treated fully relativistically and a semirelativistic approximation is used for the valence electrons. However, lead is a very heavy element and the effects of spin-orbit coupling (SOC) are expected to be considerably large. To investigate this, the spin-orbit coupling was included in a second self-consistent cycle using perturbation theory with the scalar-relativistic orbitals as basis set.

A complete cut through the surface Brillouin zone (SBZ) obtained from a film with a nominal coverage of two monolayers (2 ML) of $\mathrm{Pb}$ on graphitized $\mathrm{SiC}$, as displayed in Fig. 1 , can be obtained by combining several $k_{\|}$versus energy images. It covers $k_{\|}$space from the boundary of the Brillouin zone at $\bar{M}$ through the zone center $\bar{\Gamma}$ into the center of the next Brillouin zone, $\bar{\Gamma}^{\prime}$ (a data range of $85^{\circ}$ polar angle in electron emission). For the measurement of such welldefined features over such a wide angular range, the electrons need a large coherence length in the in-plane direction, which is an indication of the good crystalline quality of the films. This is confirmed by the sharp low-energy electron diffraction (LEED) spots (inset Fig. 1), where the inner spots originate from the $\mathrm{Pb}$ islands and the outer spots from the graphite substrate, giving a graphic representation of the large lattice mismatch between the two materials.

In a photoemission experiment, quantum well states will show up as sharp lines with distinct binding energies, depending on the film thickness. In the direction parallel to the surface, the electrons experience no confinement and the bands are expected to show a free-electron-like dispersion. At the zone center, strong emission from three quantum well states can be readily identified at $1.07 \mathrm{eV}, 1.63 \mathrm{eV}$, and $2.75 \mathrm{eV}$ binding energy; weaker features are visible at 0.65 and $2.1 \mathrm{eV}$. Taking the energy spacing between the measured QWSs and their relative intensities into account, we conclude that the lines must originate from different thicknesses; i.e., the initial layer has broken up into islands of different heights.

From a comparison with DFT calculations, two island heights are readily identified; the states at $2.75 \mathrm{eV}$ and $1.63 \mathrm{eV}$ originate from 2-ML- [blue(dark gray) line] and 4-ML- [red (light gray) line] high islands, respectively. As can be observed, the match between measurement and calculation is extremely good for the full surface Brillouin zone. The fact that the match is excellent even for a 2-ML-thick film demonstrates that the layers are quasi-free-standing, because films of low thickness typically experience strain due to the lattice structure of the substrate, which in turn influ- ences the QWS binding energy or limits their formation. Furthermore, substituting the metal-vacuum interface for a metal-substrate interface substantially alters the electron transport through this barrier and increases the effective width of the well by a fraction of a monolayer. This increase in the width of the quantum well is reflected in an increase of the binding energy of the QWSs. In $\mathrm{Pb} / \mathrm{Cu}(111)$, for example, this change in binding energy is as large as $0.3 \mathrm{eV}$ for a 5-ML-thick film. ${ }^{20}$ Also for $\mathrm{Pb} / \mathrm{Si}(111)$ this so-called phase shift has to be incorporated in the model in order to match the measured data. ${ }^{21}$

The fact that the $\mathrm{Pb}$ islands experience only a very limited influence of the substrate allows for a more elaborate comparison between experiment and theory. In Fig. 2, density functional theory (DFT) calculations for a free-standing 2-ML-thick $\mathrm{Pb}$ slab, both including [blue (dark gray) line] and ignoring spin-orbit interaction [green (light gray) line], are superimposed on an off normal photoemission image. The necessity to include SOC into the calculations is obvious; the calculations without SOC only give a poor representation of the measured data in the region of the avoided crossing between the $6 p_{z^{-}}$and $6 p_{x, y}$-derived states. (In the calculations shown in Fig. 1, SOC was already included.) This indicates the importance of these quasi-free-standing films as a test for theoretical expectations.

The QWSs can be assigned to the island heights they originate from through an analysis of the photoemission spectra as a function of total coverage. Figure 3 shows a series of energy distribution curves (EDCs) obtained by plot-

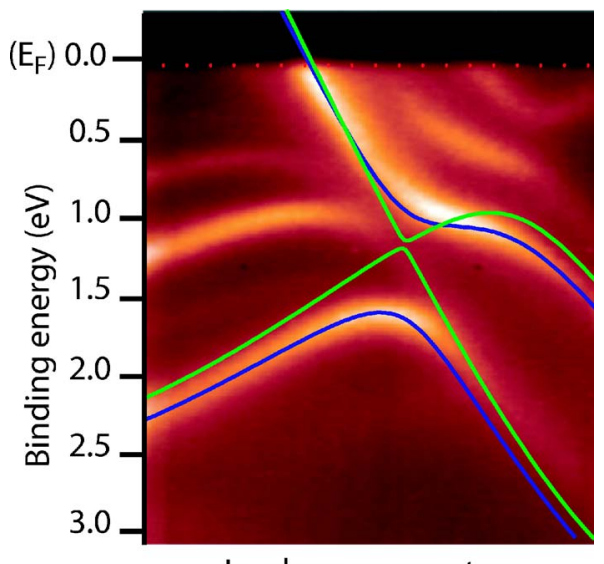

In-plane momentum

FIG. 2. (Color online) Magnified area from Fig. 1. Inserted are DFT calculations for a 2-ML-thick free-standing $\mathrm{Pb}$ incorporating [blue (dark gray) line] and ignoring spin-orbit coupling [green (light gray) line]. 


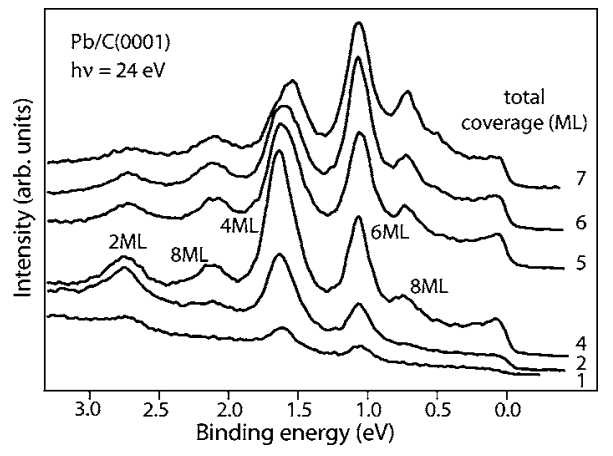

FIG. 3. Energy distribution curves extracted from the images at $k_{\|}=0$ for increasing coverage. Indicated are the island heights that the QWSs are derived from.

ting the intensity at $k_{\|}=0$ as a function of binding energy. The features present after depositing $2 \mathrm{ML}$ of $\mathrm{Pb}$, corresponding to Fig. 1, remain stationary for all higher coverages; changes occur only in the relative intensities of the quantum well states. For increasing coverages it can be assumed that features belonging to thicker islands will increase and those originating from thinner islands will decrease. This is analyzed quantitatively by fitting the QWS-derived peaks with Voigt functions on a third-degree polynomial background, as shown for the 4-ML spectrum in Fig. 4(a). From this analysis the QWS binding energies can be compared to those obtained from calculations for free-standing $\mathrm{Pb}$ layers. Moreover, the fits also yield the area underneath the individual peaks and thus the population of the different islands. Here it has to be noted that this approach to determine the island height distribution is rather indirect, but has the advantage compared to, for example, STM measurements that one determines this distribution on a macroscopic scale. Taking these considerations into account and using the binding energies obtained from calculations for free-standing $\mathrm{Pb}$ layers as a reference, we assign the QWSs to specific layer thicknesses as indicated in Fig. 3. The state with the lowest binding energy belongs to the 8-ML-high islands, the next peak to $6 \mathrm{ML}$, then $4 \mathrm{ML}$ and $8 \mathrm{ML}$, with the highest binding energy peak belonging to 2-ML-thick islands.

Figure 4(b) shows the ratio of peak area of the leading QWSs derived from the different island heights to the total peak area. This represents the fractional area that is covered by islands of a certain height. For the different island heights the trends are similar; a maximum is reached at, or close to, the respective nominal coverage. This indicates that for coverages up to at least $7 \mathrm{ML}$, only 2-, 4-, 6-, and 8-ML-high islands are formed. Thus, the even-numbered island heights are of the "preferred" type ${ }^{22}$ while the odd-numbered ones (for example 3, 5, and $7 \mathrm{ML}$ ) are unstable.

From the trends of the fractional coverage of certain island heights in Fig. 4(b) a hierarchy of stability can be determined. For low nominal coverages (1 or $2 \mathrm{ML}$ ), almost half of the Pb-covered surface consists of 4-ML-high islands, 2- and 6-ML-high islands constitute about $29 \%$ and $18 \%$, respectively, and hardly any 8-ML-high islands are found. It appears that the 4-ML-high islands are formed as long as possible; i.e., up to a nominal coverage of $4 \mathrm{ML}$ is reached. The hierarchy of stability is thus that most stable are the
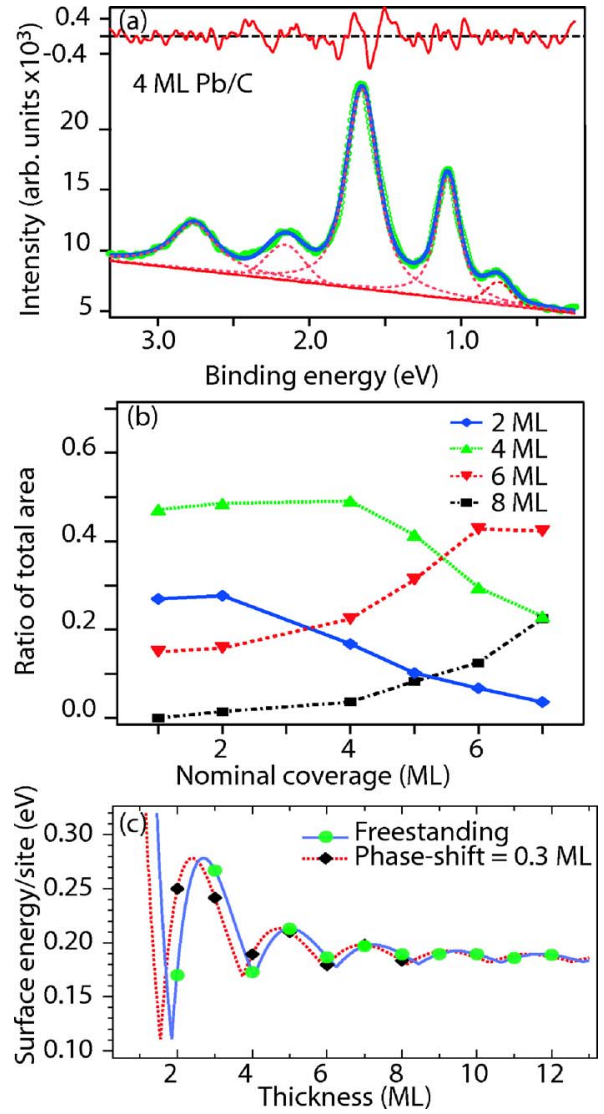

FIG. 4. (Color online) (a) Fitted spectrum for $4 \mathrm{ML} \mathrm{Pb}$ on graphite. (b) Ratio of the total $\mathrm{Pb}$ covered area for 2- [blue (dark gray) diamonds and solid line], 4- [green (light gray) upwards triangles and solid line], 6- [red (dark gray) downwards triangles and dashed line], and 8- (black squares and dashed line) ML-high islands. (c) Surface energy per unit cell as a function of layer thickness obtained with the values described in the text. The solid (blue) line and (green) circles represent a free-standing $\mathrm{Pb}$ film; for the dashed (red) line and black diamonds an artificial phase shift of 0.3 ML has been incorporated.

4-ML-high islands, followed by 2-, 6-, and finally 8-MLhigh islands.

For films to become magically stable, there has to be a local minimum in the surface energy of the layer. This surface energy can be modeled by the damped sinusoidal function ${ }^{23} E_{S}=\left[A\left|\sin \left(k_{F} d N+\varphi_{0}\right)\right|+B\right] / N^{\alpha}+C$, where $N$ is the number of ML, $d$ the interlayer spacing, and $k_{F}$ the electron wave vector at the Fermi level, measured from the $L$ point of the Brillouin zone. $A, B, C, \varphi_{0}$, and $\alpha$ are constants independent of layer height but specific for the element under investigation. The results of this equation for $\alpha=2, A=1$, $B=-0.25, C=0.184$, and $\varphi_{0}=0.5$ are depicted as the solid circles and solid line in Fig. 4(c). These values have been used to reproduce the calculated results from Ref. 23. Clear minima in the surface energy are distinguishable for 2-, 4-, and 6-ML-thick layers, which directly correspond to the stable island heights mentioned above.

In the present experiments, a major advantage of ARPES is that not only the result of electronic growth, but also its origin can be detected-thus the occurrence of islands of 2-, 
4-, 6-, and 8-ML-high $\mathrm{Pb}$ layers only, which can be reasonably well explained by a minimization of the surface energy. The reason for this becomes even more obvious when considering the full in-plane electronic structure as depicted in Fig. 1. Although the $6 p_{x y}$ bands cross the Fermi level for every given height, a crossing of the $6 p_{z}$ bands can be avoided for certain island heights. The $6 p_{z}$ bands of the QWSs formed in the magic height islands, whose electronic structure is depicted, do not come within a binding energy of several $k_{B} T$ of the Fermi level. The QWS from the 8-MLhigh islands is only faintly visible, but remains below $E_{\mathrm{F}}$. The QWSs formed for the intermediate coverages all have $6 p_{z}$ bands that cross the Fermi level and are therefore suppressed.

Considering the magic heights for $\mathrm{Pb} / \mathrm{C}$ and the energy variations shown by the solid line in Fig. 4(c), the question arises why the 2-ML-thick islands have not been previously observed for thin $\mathrm{Pb}$ layers and the 4-ML-thick islands only when a third species (In) is placed at the interface. ${ }^{24}$ Over the full range of $k$, their bands are further below the Fermi level than those of the 6-ML islands, which are observed to be exceptionally stable on the $\sqrt{3}$ reconstruction of $\mathrm{Si}(111)$. Also, the energy minima in Fig. 4(c) are much deeper at 2 and $4 \mathrm{ML}$ than at a thickness of $6 \mathrm{ML}$. It is quite likely that for $\mathrm{Si}(111)$, charge transfer across the interface is much larger than for vacuum or graphite, or in other words the phase shift at the interface will be larger. This will increase the effective width of the well and therefore the value of $N$, and the entire damped sinusoidal function will shift to lower coverages, as indicated by the (red) dashed line and diamonds for a phase shift of $0.3 \mathrm{ML}$. The curve for the freestanding film already has a high positive slope at 2 and 4 ML, and the surface energy thus increases strongly. On the other hand, at $6 \mathrm{ML}$ the sinusoidal function has a negative slope, and a slightly larger phase shift will thus lower the surface energy. A second explanation is based on the fact that $\mathrm{Pb}$ and $\mathrm{Si}$ have a $9 \%$ lattice mismatch (based on the bulk crystal structure) and that, unlike for $\mathrm{Pb}$ on graphite, the $\mathrm{Pb}$ overlayer lattice structure is influenced by that of the $\mathrm{Si}(111)$. This induces a large amount of strain in the $\mathrm{Pb}$ layers. This strain is larger for thinner films, therefore increasing the surface energy of the 2- and 4-ML-high islands, but hardly affecting the thicker films.

This work was supported as part of the European Science Foundation EUROCORES program SONS under program MOL-VIC through funds from the Deutsche Forschungsgemeinschaft and the EC Sixth Framework program. J.H.D. acknowledges support by the International Max-Planck Research School "Complex Surfaces in Material Sciences." We gratefully acknowledge support by G. Reichardt and the BESSY staff.
${ }^{1}$ T.-C. Chiang, Surf. Sci. Rep. 39, 181 (2000).

${ }^{2}$ M. Milun, P. Pervan, and D. P. Woodruff, Rep. Prog. Phys. 65, 99 (2002).

${ }^{3}$ M. Jalochowski, M. Hoffmann, and E. Bauer, Phys. Rev. Lett. 76, 4227 (1996).

${ }^{4}$ I. Vilfan, M. Henzler, O. Pfennigstorf, and H. Pfnür, Phys. Rev. B 66, 241306(R) (2002).

${ }^{5}$ L. Aballe, A. Barinov, A. Locatelli, S. Heun, and M. Kiskinova, Phys. Rev. Lett. 93, 196103 (2004).

${ }^{6}$ B. G. Orr, H. M. Jaeger, and A. M. Goldman, Phys. Rev. Lett. 53, 2046 (1984).

${ }^{7}$ Y. Guo et al., Science 306, 1915 (2004).

${ }^{8}$ K. Budde, E. Abram, V. Yeh, and M. C. Tringides, Phys. Rev. B 61, R10602 (2000); D. Luh, T. Miller, J. J. Paggel, M. Y. Chou, and T.-C. Chiang, Science 292, 1131 (2001).

${ }^{9}$ J. J. Paggel, D.-A. Luh, T. Miller, and T.-C. Chiang, Phys. Rev. Lett. 92, 186803 (2004).

${ }^{10}$ Z. Zhang, Q. Niu, and C. K. Shih, Phys. Rev. Lett. 80, 5381 (1998).

${ }^{11}$ V. Yeh, L. Berbil-Bautista, C. Z. Wang, K. M. Ho, and M. C. Tringides, Phys. Rev. Lett. 85, 5158 (2000).

${ }^{12}$ R. Otero, A. L. Vazquez de Parga, and R. Miranda, Phys. Rev. B 66, 115401 (2002).
${ }^{13}$ P. M. Echenique and J. B. Pendry, J. Phys. C 11, 133 (1978).

${ }^{14}$ I. Forbeaux, J.-M. Themlin, and J.-M. Debever, Phys. Rev. B 58, 16396 (1998).

${ }^{15}$ J. Bernhardt, J. Schardt, U. Starke, and K. Heinz, Appl. Phys. Lett. 74, 1084 (1999).

${ }^{16}$ Th. Seyller et al., Surf. Sci. 600, 3845 (2006).

${ }^{17}$ K. Horn, B. Reihl, A. Zartner, D. E. Eastman, K. Hermann, and J. Noffke, Phys. Rev. B 30, 1711 (1984).

${ }^{18}$ J. H. Dil, J. W. Kim, S. Gokhale, M. Tallarida, and K. Horn, Phys. Rev. B 70, 045405 (2004).

${ }^{19}$ J. P. Perdew, K. Burke, and M. Ernzerhof, Phys. Rev. Lett. 77, 3865 (1996).

${ }^{20}$ K. Schwarz and P. Blaha, Comput. Mater. Sci. 28, 259 (2003).

${ }^{21}$ M. H. Upton, C. M. Wei, M. Y. Chou, T. Miller, and T.-C. Chiang, Phys. Rev. Lett. 93, 026802 (2004).

${ }^{22}$ Although the derivation is from layers that were not annealed, due to the low diffusion barrier for $\mathrm{Pb}$ on graphite even at $100 \mathrm{~K}$, the resulting island heights can be regarded as preferred heights.

${ }^{23}$ C. M. Wei and M. Y. Chou, Phys. Rev. B 66, 233408 (2002).

${ }^{24}$ M. Hupalo and M. C. Tringides, Phys. Rev. B 73, 041405(R) (2006). 